\title{
Melatoninergic Antidepressant in Post-Stroke Depression Therapy
}

\author{
Victor V. Kuznetsov \\ Department of Cerebral Vascular Pathology, Chebotarev Institute of Gerontology, National Academy of Medical Sciences, Kiev, \\ Ukraine \\ Email: stroke@geront.kiev.ua
}

How to cite this paper: Kuznetsov, V.V. (2018) Melatoninergic Antidepressant in Post-Stroke Depression Therapy. Advances in Aging Research, 7, 29-38. https://doi.org/10.4236/aar.2018.72003

Received: November 6, 2017

Accepted: March 26, 2018

Published: March 29, 2018

Copyright (c) 2018 by author and Scientific Research Publishing Inc. This work is licensed under the Creative Commons Attribution International License (CC BY 4.0). http://creativecommons.org/licenses/by/4.0/ Open Access

\begin{abstract}
This paper presents the results of the effects of Melitor $(25 \mathrm{mg}$ during two months) on the psychic-emotional state, cerebral hemodynamic and bioelectrical activity of the brain in 30 patients (average age $66.2 \pm 3.2$ years) with ischemic post-stroke depression. It has been found that this drug possesses a multimodal action which is characterized by the decrease of the level of depression, widening of the range of social-environmental activity, improvement of the cerebral blood circulation and harmonization of the brain electrogenesis. In the patients with right versus left hemisphere stroke, the effect of Melitor on the functional state of the brain was more pronounced. Based on the results obtained in our study, we can recommend Melitor to use it in complex scheme of rehabilitation care of patients of the given nosological category.
\end{abstract}

\section{Keywords}

Ischemic Stroke, Post-Stroke Depression, Cerebral Hemodynamic, Brain Bioelectrical Activity, Melitor

\section{Introduction}

Rehabilitation care of the patients after stroke is an important medico-social problem. Stroke causes motor, sensitive and psychic-emotional disturbances and narrows the range of their social and daily living activities. Long-term and systematic rehabilitation has the potential to restore the walking and self-servicing abilities [1] [2]. Among the factors negatively influencing rehabilitation of the post-stroke patients were the changes in psychic-emotional sphere, depression and dementia [3] [4]. One of the main mechanisms of a negative impact of depressive disorders on the effective recovery treatment is lower patient's motiva- 
tion in participation in the rehabilitation measures [5].

Prevalence of the post-stroke depression varies within $40 \%-50 \%$. Women are more affected with vascular depression. Its peak is seen during first 3 - 6 months after stroke. However, the risk of depression appearance remains high during first two years [6] [7]. The risk factors of post-stroke depression are thought to be the age, prestroke subcortical atrophy, family and personal history indicating psychiatric disorders in the past [8] [9]. It has been found that post-stroke depression is strongly correlated with cognitive impairments, passive perception of the quality of life and increased mortality [10] [11] [12]. According to the transcranial doplerography, the cerebral blood flow velocity is reduced in the patients with post-stroke depression. Therefore, adequate treatment of post-stroke depression should be considered not only as the way of reducing depressive symptoms, but also as the way of improving life quality, enhancing rehabilitation care efficacy and reducing physical disorders [13]. In recent years, it has been reported that certain groups of antidepressants, when administered during acute post-stroke period, enhance brain plasticity and thereby promote maximal neurological recovery after stroke.

Drug therapy of post-stroke depression is considered the main part of rehabilitation care of patients after stroke [14] [15]. The main approach of present-day treatment of post-stroke depression is the prescription of antidepressants [16]. Perfection of approaches towards post-stroke depression treatment is tightly linked with the development of monoamine hypothesis of depressive conditions due to serotonin and noradrenaline deficit in the synaptic chain slot [17]. In the treatment of post-stroke depression, the most frequently used is the selective serotonin reuptake inhibitors (SSRIs). This class of antidepressants causes fewer side effects in the comparison with tricyclic antidepressants which often cause the cardiotoxic reactions. However, the use of SSRIs is also limited because of the population of patients with cerebral-vascular pathology refractory to SSRIs therapy [18].

The new generation of antidepressants, melatoninergic antidepressants in particular, is the first choice for post-stroke depression therapy. The representative of this class is Agomelatine developed by the pharmaceutical company Servier. Melitor is an antidepressant which is capable of renewing circadian rhythms being disturbed in the patients with depression. Melitor is the agonist of MT-1 and MT-2 melatonin receptors and the antagonist of serotonin 5- $\mathrm{HT}_{2 \mathrm{c}}$ receptors located in the suprachiasmatic nucleus and regulating circadian rhythms. Influence of Melitor on these three types of the receptors ensures a marked antidepressant and anxiolytic effect. Therapeutic effects of the Melitorare seen by the end of the first week of treatment, when the circadian rhythm of the patients normalized, his daily activity and sleep are improved. His spirit and cognitive functions become improved over the two-three week period [19].

Aim: Complex analysis of influences of the Melitor Agomelatine on the functional state of the CNS in the patients with post-stroke depression. 


\section{Patients and Methods}

Thirty elderly patients (six months after acute period) with thrombolytic stroke took part in the study. They had the diagnosis of post-stroke depression in the carotid basin $(20.2 \pm 1.4$ scores according to the Hamilton scale and $12.8 \pm 1.2$ according to the gerontological scale). The patients were given the drug, Melitor, in a single 25-mg dose for the night during two months. Before and after Melitor intake we performed complex examination including neuropsychological examinations (gerontological depression scale, Hamilton scale), measurement of social-daily living activity (Bartel index), cerebral hemodynamic (EnVisor, Philips) and brain bioelectrical activity (Neurofax EEG-1100 K, Nihon Kohden). Data were statistically analyzed using Statistica ${ }^{\oplus}$ for Windows 6.0. The presence of correlations between cerebral hemodynamic and the power of the main EEG rhythms was found by assessing statistically significant correlation coefficients $(r \geq 0.70)$.

\section{Results}

Our analysis of the dynamic of subjective complaints of the post-stroke patients with depression before and after a two-month Melitor course treatment has showed the reduced number and intensity of their complaints (Table 1).

After two months of Melitor treatment there was an improvement of sleep, reduced physical fatigue during exercising, less frequent headaches and irritability in the patients with both, left- and right-sided stroke.

The levels of depression and anxiety were reduced after Melitor treatment (Table 2).

In the patients with left-hemispheric stroke we observed the statistically significant decrease of the indices of constitutional anxiety according to the Spilberg-Khaninscale $(46.7 \pm 2.1$ to $38.1 \pm 1.8$ scores before and after treatment, respectively) and the indices of reactive anxiety were also decreased (from $44.6 \pm$ 2.2 to $34.2 \pm 1.1$ scores). At the right-sided ischemia the level of constitutional and reactive anxiety were also reduced: from $51.5 \pm 2.0$ to $41.4 \pm 2.31$ and from $47.1 \pm 1.8$ to $37.0 \pm 3.1$ scores, respectively.

Table 1. Dynamic of subjective complaints of post-stroke patients before and after a two-month Melitor course treatment, \%.

\begin{tabular}{ccccc}
\hline \multirow{2}{*}{ Complaints } & \multicolumn{2}{c}{ Right hemisphere is chemia } & \multicolumn{2}{c}{ Left hemisphere ischemia } \\
\cline { 2 - 5 } & Before treatment & After treatment & Before treatment After treatment \\
\hline Bad sleep & 46.7 & 16.7 & 43.3 & 20 \\
Attention disorders & 40 & 20 & 40 & 16.7 \\
Appetite disorders & 43.3 & 26.7 & 40 & 23.3 \\
Internal strain & 50 & 16.7 & 46.7 & 20 \\
Headaches & 50 & 20 & 43.3 & 23.3 \\
Physical fatigue & 50 & 20 & 46.7 & 16.7 \\
\hline
\end{tabular}

*-Statistically significant differences before and after treatment. 
Table 2. Dynamic of the indices of Hamilton and gerontological scales in the post-stroke patients before and after Melitor treatment, scores.

\begin{tabular}{|c|c|c|c|c|}
\hline \multirow[b]{2}{*}{ Scales } & \multicolumn{2}{|c|}{ Right hemisphere ischemia } & \multicolumn{2}{|c|}{ Lefthemisphere ischemia } \\
\hline & $\begin{array}{c}\text { Before } \\
\text { treatment }\end{array}$ & After treatment & Before treatment & After treatment \\
\hline Gerontological scale & $15.1 \pm 1.3$ & $7.1 \pm 1.8^{*}$ & $11.2 \pm 1.2$ & $4.3 \pm 1.2^{*}$ \\
\hline Hamilton scale & $21.0 \pm 1.5$ & $8.2 \pm 1.05^{\star}$ & $19.0 \pm 1.2$ & $7.4 \pm 1.0^{*}$ \\
\hline
\end{tabular}

*-Statistically significant differences before and after treatment.

Thus the Melitor produces the anxyolytic effect, namely reduced anxiety among depressive disturbances. According to the Barthel scale measurements, the levels of social and daily living activity increased from $78.3 \pm 2.4$ to $91.5 \pm 1.8$ scores. Clinically this was shown by increased motor activity and wider range of daily living self-servicing and better coordination.

To analyze the mechanisms determining Melitor effects on the psychic-emotional condition of post-stroke patients, we performed analyses of the brain functioning (according to the indices of cerebral blood circulation and bioelectric activity of the brain) before and after course of treatment with Melitor. We observed improvements in cerebral hemodynamic, judging by the increase of systolic linear speed of blood flow (LSBF) in separate parts of the carotid and vertebral-basilar basins.

There is however another remark to the drug effect, it has shown the hemispheric peculiarities on the cerebral blood speed (Figure 1, Table 3).

In the patients with right versus left hemisphere stroke, the hemodynamic effect was more marked. With right-sided ischemia localization, the statistically significant increase of LSBF rate was observed in the right MCA, left ICA, in the two VA, and in the two ACA and BA. With left-hemispheric ischemia localization there was a statistical increase of LSBF only in BA.

In the post-stroke patients the course treatment with Melitor brought about the decrease of the peripheral resistance predominantly in the patients with left hemisphere localization. Thus in the patients with right hemisphere stroke the pulsatility index in the intact MCA decreased from $0.8 \pm 0.05$ before to $0.7 \pm 0.02$ after treatment, respectively. Whereas in the patients with left hemisphere stroke the pulsatility index in the injured ICA decreased from $0.9 \pm 0.04$ before to $0.8 \pm$ 0.03 after treatment, respectively; the circulatory resistance index in the injured PCA decreased from $0.5 \pm 0.02$ before to $0.4 \pm 0.03$ after treatment, respectively, that evidences for a more marked positive effect of the Melitoron the indices of the peripheral resistance in the patients with left-sided hemisphere.

Knowing that computer EEG allows assess quantify the degree and structure of the brain functional changes and their dynamic that may serve an objective criterion for assessment of the mechanisms of action of drugs in the stroke patients, we analyzed the bioelectric activity of the brain in the patients with post-stroke depression who had undergone treatment with Melitor. It appeared the structure of rearrangement of the intensity of the main EEG rhythms. 
Table 3. Dynamic of systolic linear blood flow velocity (LBFV) in the patients after stroke after course Melitor treatment, $\mathrm{cm} / \mathrm{sec}$.

\begin{tabular}{cccccc}
\hline \multirow{2}{*}{ Vessels } & \multicolumn{2}{c}{ Right hemisphere ischemia } & \multicolumn{2}{c}{ Lefthemisphere ischemia } \\
\cline { 3 - 6 } & & Before treatment & After treatment & Before treatment & After treatment \\
\hline \multirow{2}{*}{ CCA } & Injured & $61.9 \pm 4.3$ & $64.2 \pm 4.5$ & $74.5 \pm 4.5$ & $72.1 \pm 3.2$ \\
& Intact & $75.0 \pm 5.2$ & $73.1 \pm 5.5$ & $66.3 \pm 5.6$ & $69.9 \pm 4.6$ \\
\multirow{2}{*}{ ICA } & Injured & $75.5 \pm 2.5$ & $70.7 \pm 2.3$ & $72.2 \pm 7.6$ & $73.7 \pm 5.5$ \\
& Intact & $61.9 \pm 1.7$ & $70.8 \pm 2.2^{*}$ & $63.8 \pm 2.8$ & $65.6 \pm 2.6$ \\
\multirow{2}{*}{ VA } & Injured & $36.1 \pm 1.5$ & $42.7 \pm 1.9^{*}$ & $44.1 \pm 2.2$ & $44.9 \pm 2.3$ \\
& Intact & $38.2 \pm 1.9$ & $45.7 \pm 2.3^{*}$ & $39.8 \pm 1.1$ & $42.5 \pm 2.1$ \\
\multirow{2}{*}{ ABA } & Injured & $80.8 \pm 2.2$ & $87.0 \pm 2.5^{*}$ & $94.6 \pm 4.7$ & $95.2 \pm 5.3$ \\
& Intact & $75.2 \pm 2.0$ & $87.7 \pm 2.1^{*}$ & $94.2 \pm 5.7$ & $88.4 \pm 4.9$ \\
\multirow{2}{*}{ MCA } & Injured & $87.9 \pm 4.1$ & $103.7 \pm 4.5^{*}$ & $92.7 \pm 5.2$ & $94.5 \pm 5.5$ \\
& Intact & $106.0 \pm 4.8$ & $104.9 \pm 3.5$ & $107.0 \pm 4.1$ & $107.9 \pm 6.4$ \\
& Injured & $46.4 \pm 1.4$ & $52.7 \pm 2.9^{*}$ & $52.5 \pm 2.2$ & $52.8 \pm 3.1$ \\
PCA & Intact & $49.0 \pm 1.3$ & $53.7 \pm 3.1$ & $54.6 \pm 2.1$ & $54.6 \pm 2.3$ \\
& & $53.2 \pm 2.4$ & $61.0 \pm 3.4^{*}$ & $54.1 \pm 3.1$ & $61.9 \pm 2.5^{*}$ \\
\hline \multirow{2}{*}{ BA } & & & & &
\end{tabular}

*-Statistically significant differences before and after treatment.
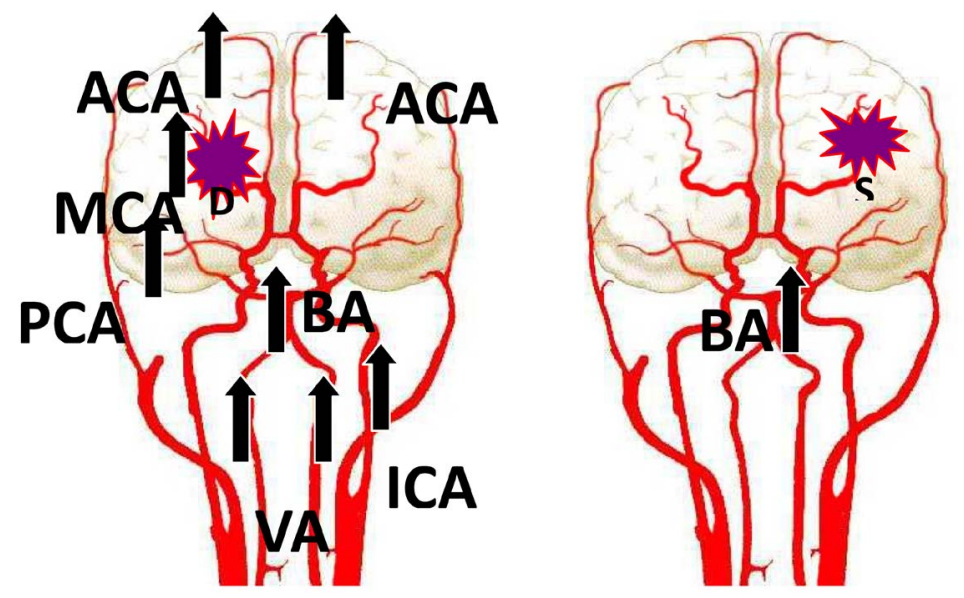

Figure 1. Changes of the LBFV in the vessels of the carotid and vertebra-basilar basins in the patients with post-stroke depression after Melitor treatment.

Figure 2 shows that the power of the delta-rhythm in all areas of the hemisphere decreased in the patients with right-sided stroke: maximally in the lobe area of the injured hemisphere (from $1.2 \pm 0.01$ before treatment to $0.8 \pm 0.07$ $\mu \mathrm{V}$ ) and of the theta-rhythm (maximally in the central area of the injured hemisphere (from $1.2 \pm 0.01$ before to $0.9 \pm 0.01 \mu \mathrm{V}$ against a background of an increased power of the alpha-1-rhythm (maximally in the central area of the injured hemisphere: from $0.9 \pm 0.02$ and $1.6 \pm 0.03 \mu \mathrm{V}$ respectively) and the frequency of the alpha-rhythm: maximally in the central area of intact hemisphere: $9.8 \pm 0.03$ 

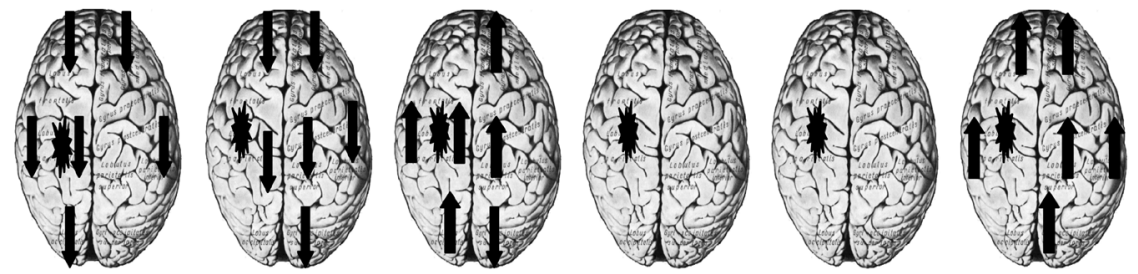

(a)
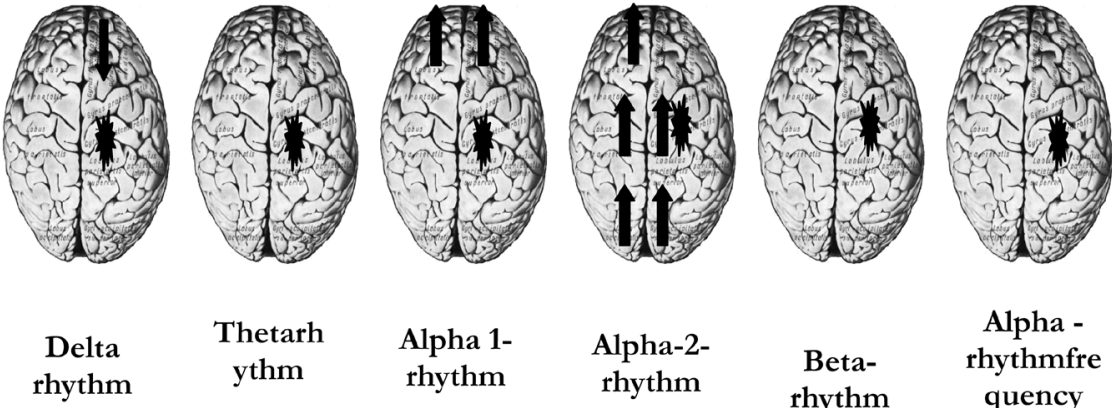

$$
\begin{gathered}
\text { Thetarh } \\
\text { ythm }
\end{gathered}
$$
Alpha 1- rhythm

Beta-
rhythm

\begin{abstract}
Alpha rhythmfre quency
\end{abstract}

(b)

Figure 2. Statistically significant changes of the power of the main EEG rhythms in the post-stroke patients with right (a) and left (b) hemisphere localization after Melitor treatment.

$\mathrm{Hz}$ before and $10.5 \pm 0.02 \mathrm{~Hz}$ after treatment. In the patients with left-hemispheric stroke the power increased only in the alpha rhythm range: maximum in the frontal area of intact hemisphere: $1.1 \pm 0.01 \mu \mathrm{V}$ before and $1.5 \pm 0.01 \mu \mathrm{V}$ after Melitor treatment and in the alpha-2 rhythm: maximum in the central area of the injured hemisphere: $0.6 \pm 0.01 \mu \mathrm{V}$ and $1.5 \pm 0.04 \mu \mathrm{V}$ respectively in both hemispheres. In the patients with post-stroke depression there also occurred the rearrangement of inter systemic relationships between cerebral hemodynamic and bioelectric activity of the brain. The type of rearrangement demonstrated marked hemispheric peculiarities (Figure 3 ).

Figure 3 shows that the structure of correlations between the power of the main EEG rhythms and indices of cerebral circulation before and after Melitor treatment in the patients with right-sided stroke quantitatively and qualitatively differed from the structure of correlations in the patients with left-sided stroke.

In the patients suffering from depression and stroke localization in the right hemisphere, the number of correlations between the power in delta and theta rhythms after Melitor treatment increased from 3 to 9 and between the power in the alpha- 1 and alpha- 2 rhythms from 1 to 3 . The total number of correlations was 4 before treatment and 12 after treatment.

In the patients suffering from depression and stroke localization in left hemisphere the range of correlations between the power of the main EEG rhythms and indices of cerebral hemodynamic was narrowed. Before Melitor treatment there were seven statistically significant correlations between the power of slow rhythms (delta and theta) and LSBF in the carotid basin vessels. After drug treatment all correlation sleveled. In the cases of left-sided stroke, 
Theta rhythmDelta rhythmAlpha-1 rhythm
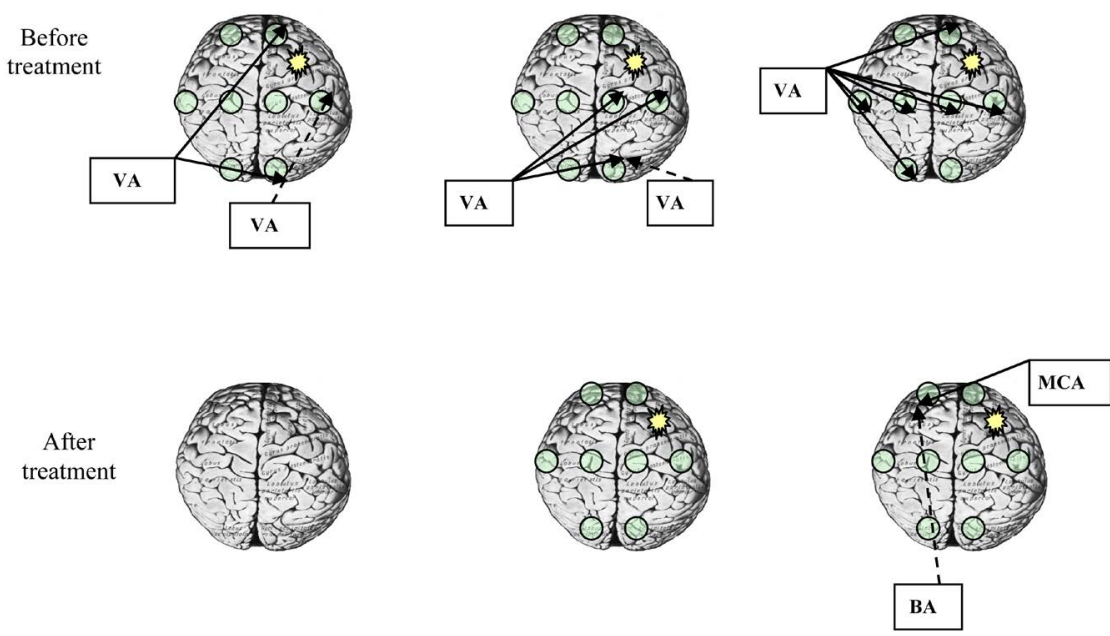

(a)
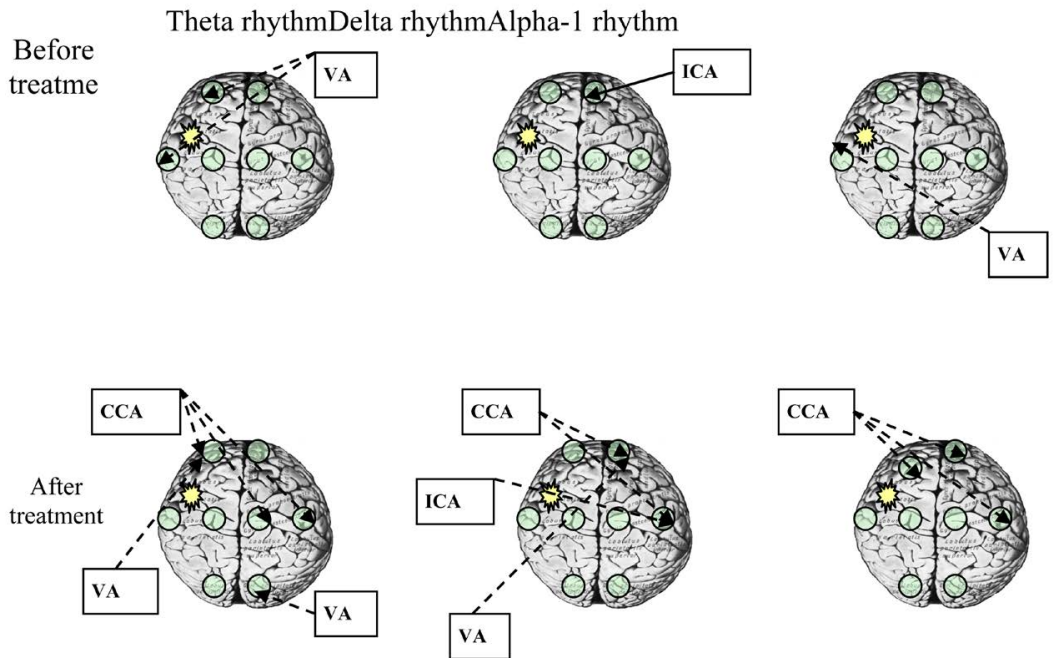

(b)

Figure 3. The structure of changes in the correlations between the LSBF in the vessels of the carotid and vertebra-basilar basins and the power of the main EEG rhythms in the patients with depression in the left hemisphere (a) and in the right hemisphere (b)) stroke after Melitor treatment.

The number of correlations betweenalpha-1rhythm power and LSBF in the vessels of carotid and vertebra-basilar basins was six. After Melitor treatment there were only two. The total number of correlations was 13 before treatment and 2 after treatment.

Summing up, owing to Melitor treatment of patients with ischemic stroke we observed the decrease of anxiety and depression, the expansion of functional activity range, the improvement of cerebral hemodynamic and functional-metabolic processes in the structures generating bioelectric activity of the brain as well as reorganization of intersystemic relationships between cerebral hemodynamic and bioelectric activity of the brain. The drug, Melitor, has produced a more marked 
influence on the psychic-emotional activity, cerebral hemodynamic and bioelectric activity of the brain in the patients with the right hemisphere stroke.

\section{Conclusions}

Benefits of the two-month treatment of the patients with post-stroke depression using the medication Melitor in the $25 \mathrm{mg}$ dose daily are as follows:

1) Overall health improvement, better sleep, lesser frequency and intensity of headaches, lower level of anxiety depressivity, wider range of social-daily living activities.

2) With right hemisphere stroke, the cerebral blood flow increased in separate vessels of the carotid (MCA, PCA and ICA) and vertebral-basilar (VA) basins. With left hemisphere stroke, there was a statistically significant increase of LBFV only in the vertebral-basilar basin vessels.

3) In the patients with depression and right hemisphere stroke, the power in the range of slow rhythms was decreased against the background increased power in the alpha rhythm.

4) The patients with left hemisphere stroke displayed an increase of the power only in the alpha rhythm range.

5) With right-versus left-hemisphere stroke localization, there was a more marked drug effect on the cerebral hemodynamic and bioelectrical activity of the brain.

In the left hemisphere, the range of correlations between LBFV and the intensity of separate EEG rhythms was six times reduced whereas with the right-sided stroke, it was three times increased that evidenced for the morpho-functional and biochemical asymmetry of the brain.

\section{References}

[1] Gusev, E.I. and Skvortsova, V.I. (2001) Brain Ischemia. Meditsina Publishers, Moscow.

[2] Jeong, Y.J., Kim, W.C., Kim, Y.S., Choi, K.W., Son, S.Y. and Jeong, Y.G. (2014) The Relationship between Rehabilitation and Changes in Depression in Stroke Patients. Journal of Physical Therapy Science, 26, 1263-1266.

[3] Douven, E., Kohler, S., Schievink, S.H.J., van Oostenrugge, R.J., Staals, J., Verhey, F.R.J. and Aalten, P. (2017) Temporal Associations between Fatigue, Depression and Apathy after Stroke: Results of the Cognition and Affect after Stroke: A Prospective Evaluation of Risks Study. Cerebrovascular Diseases, 44, 330-337. https://doi.org/10.1159/000481577

[4] Kevin, D., Niedens, M. and Wilson, J. (2013) Treatment Outcomes of a Crisis Intervention Program for Dementia with Severe Psychiatric Complications: The Kansas Bridge Project. The Gerontologist, 53, 102-112. https://doi.org/10.1093/geront/gns104

[5] Kuznetsov, V.V. and Glushko, S.F. (2013) Cerebral Hemodynamic in the Patients with Post-Stroke Depression. Psychic Health, 2, 11-17.

[6] Hackett, M.L., Anderson, C.S. and House, A.O. (2005) Management of Depression after Stroke: A Systematic Review of Pharmacological Therapies. Stroke, 36, 1098-1103. 
https://doi.org/10.1161/01.STR.0000162391.27991.9d

[7] Smulevych, A.B. (2007) Depression at Somatic and Psychic Diseases. Medical Inform Agency, Moscow.

[8] Kim, J.S. (2016) Post-Stroke Mood and Emotional Disturbances: Pharmacological Therapy Based on Mechanisms. Journal of Stroke, 18, 244-255. https://doi.org/10.5853/jos.2016.01144

[9] Wein, A.M., Voznesenskaya, T.G., Golubev, V.L. and Dyukova, G.M. (2007) Depression in Neurologic Practice: Clinic, Diagnosis, Treatment. Medical Inform Agency, Moscow.

[10] Emerski, E. and Robinson, R. (2000) The Neuropsychiatry of Stroke. Psychosomatics, 41, 5-14. https://doi.org/10.1016/S0033-3182(00)71168-6

[11] Gainotti, G., Antonucci, G., Marra, C. and Paolucci, S. (2001) Relation between Depression after Stroke, Antidepressant Therapy and Functional Recovery. Journal of Neurology and Neurosurgical Psychiatry, 71, 258-261.

https://doi.org/10.1136/jnnp.71.2.258

[12] Verdelho, A., Henon, H., Lebert, F., Pasquier, F. and Leys, D. (2004) Depressive Symptoms after Stroke and Relationship with Dementia. A Three-Year Follow-Up Study. Neurology, 62, 905-911. https://doi.org/10.1212/01.WNL.0000115107.66957.8C

[13] Kasper, S., Hale, A. and Lemoine, P. (2008) Depression after Stroke. Journal of the European College of Neuropsychopharmacology, 19, 412-413. https://doi.org/10.1016/S0924-977X(09)70638-8

[14] Pankaj, Sh. and Meschia, J.F. (2013) Stroke Genetics. Springer Verlag, London.

[15] Annoni, J.M., Staub, F., Bruggimann, L., Gramigna, S. and Bogousslavsky, J. (2006) Emotional Disturbances after Stroke. Clinical Experimental Hypertension Journal, 28, 243-249. https://doi.org/10.1080/10641960600549173

[16] Geyer, J.D. and Gomez, C.R. (2009) Stroke: A Practical Approach. Lippincott Williams \&Wilkins.

[17] Robinson, R.G. and Spalletta, G. (2010) Poststroke Depression: A Review. Canadian Journal of Psychiatry, 55, 341-349. https://doi.org/10.1177/070674371005500602

[18] Bartoli, F., Lillia, N., Lax, A., Crocamo, C., Mantero, V., Carra, G., Agostoni, E. and Clerici, M. (2013) Depression after Stroke and Risk of Mortality: A Systematic Review and Meta-Analysis. Stroke Research and Treatment, 862-978.

[19] Kuznetsova and Glushko, S.F. (2012) Therapy of Post-Stroke Depression: Melatoninergic Antidepressants. Health in Ukraine, 2-3. 


\section{Abbreviations}

ACA: Anterior Cerebral Artery;

BA: Basilar Artery;

CCA: Common Carotid Artery;

ICA: Internal Carotid Artery;

MCA: Middlecerebral Artery;

PCA: Posterior Cerebral Artery;

VA: Vertebral Artery;

LSBF: Linear Speed of Blood Flow;

SSRIs: Selective Serotonin Reuptake Inhibitors. 